\title{
A Finite Memory Structure Smoother with Recursive Form Using Forgetting Factor
}

\author{
Pyung Soo Kim \\ Department of Electronic Engineering, Korea Polytechnic University, 237 Sangidaehak-Ro, Siheung-Si, \\ Gyeonggi-Do 15073, Republic of Korea \\ Correspondence should be addressed to Pyung Soo Kim; pspeter.kim@gmail.com
}

Received 17 March 2017; Accepted 25 May 2017; Published 19 June 2017

Academic Editor: Alessandro Mauro

Copyright (C) 2017 Pyung Soo Kim. This is an open access article distributed under the Creative Commons Attribution License, which permits unrestricted use, distribution, and reproduction in any medium, provided the original work is properly cited.

\begin{abstract}
This paper proposes an alternative finite memory structure (FMS) smoother with a recursive form under a least squares criterion using a forgetting factor strategy. The proposed FMS smoother does not require information of the noise covariances as well as the initial state. The proposed FMS smoother is shown to have good inherent properties such as time-invariance, unbiasedness, and deadbeat. The forgetting factor is shown to be considered as useful parameter to make the estimation performance of the proposed FMS smoother as good as possible. Through computer simulations for the F-404 engine system, it is shown that the proposed FMS smoother can be better than the existing FMS smoother for incorrect noise covariances and the IMS smoother for temporary uncertainties.
\end{abstract}

\section{Introduction}

Recently, to overcome the resulting problems of existing fixed-lag Kalman smoother with the infinite memory structure (IMS) in [1-5], the fixed-lag smoother with the finite memory structure (FMS) has been developed for state estimation in discrete time-invariant systems [6-9] and discrete time-varying systems [10]. This FMS smoother has been known to have some good properties such as unbiasedness and deadbeat, which cannot be obtained by the IMS smoother. Moreover, in contrast to the IMS smoother with the recursive structure that tends to accumulate the smoothing error with the progression of time, the FMS smoother is inherently bounded input/bounded output stable and more robust against temporarily uncertain model parameters and round-off errors due to the FMS as shown in [11-15].

However, existing FMS smoothers in [6-10] have some limitations. Firstly, information about noise covariances to obtain the optimal estimate should be also assumed to be exactly known as the IMS smoother, which may be somewhat restrictive. Secondly, the recursive form of the FMS smoother has not been developed. Thus, for a large system dimension or window length, the computation load may be burdensome in real-time computation.
In this paper, to overcome the resulting difficulties in applications of existing FMS smoother, an alternative FMS smoother with a recursive form is proposed under a least squares criterion using a forgetting factor strategy. The forgetting factor strategy has been well-known in estimation areas to give exponentially less weight to older error samples as shown in [16-18]. The proposed FMS smoother does not require a priori information about noises covariance as well as the initial state. The proposed FMS smoother has good inherent properties such as time-invariance, unbiasedness, and deadbeat. The proposed FMS smoother is represented in a recursive form as well as a matrix form. It is noted that the recursive form has not been developed by the existing FMS smoother in [6-10]. The forgetting factor is shown to be considered as useful parameter to make the estimation performance of the proposed FMS smoother as good as possible. Finally, extensive computer simulations are performed for the F-404 engine system. As would be expected, the existing FMS smoother with correct noise covariances outperforms the proposed FMS smoother, since the existing FMS smoother is an optimal state estimate under the correct noise covariances. However, in comparison with the existing FMS smoother with incorrect noise covariances, the proposed FMS smoother can be better. In addition, the 
proposed FMS smoother is shown to be better than the IMS smoother for temporary uncertainties.

This paper is organized as follows. In Section 2, the FMS estimation for state-space model is reviewed briefly. In Section 3, an alternative FMS smoother is proposed and its inherent properties are shown. In Section 4, extensive computer simulations are performed to verify the proposed FMS smoother. Finally, conclusions are presented in Section 5.

\section{Finite Memory Structure Estimation for State-Space Model}

A discrete time state-space model is considered as follows:

$$
\begin{gathered}
x(i+1)=A x(i)+G w(i), \\
z(i)=C x(i)+v(i),
\end{gathered}
$$

where $x(\cdot)\left(\in \mathfrak{R}^{n}\right)$ is the state and $z(\cdot)\left(\in \mathfrak{R}^{q}\right)$ is the measurement. The system noise $w(\cdot)\left(\in \mathfrak{R}^{p}\right)$ and the measurement noise $v(\cdot)\left(\in \mathfrak{R}^{q}\right)$ are zero-mean white Gaussian and mutually uncorrelated. The covariances of $w(\cdot)$ and $v(\cdot)$ are denoted by $Q$ and $R$, respectively.

In this paper, for the state estimation in the stochastic state-space models (1), the finite memory structure (FMS) is considered. The FMS estimation utilizes only the finite number of measurements on the most recent interval $[i-$ $M, i]$ called the window and discards past measurements outside the window. This window of finite measurements recedes forward in time at each sampling time when a new measurement is available.

The finite measurements on the most recent window $[i-$ $M, i]$ are denoted by $Z(i)$ as follows:

$$
Z(i) \triangleq\left[\begin{array}{lllll}
z^{T}(i-M) & z^{T}(i-M+1) & \cdots & z^{T}(i-1)
\end{array}\right]^{T}
$$

and can be represented in the following regression form from the discrete time state-space model (1):

$$
Z(i)=L x(i-M)+N W(i)+V(i),
$$

where $W(i), V(i)$ have the same form as (2) and matrices $L$, $N$ are as follows:

$$
\begin{gathered}
L \triangleq\left[\begin{array}{c}
C \\
C A \\
\vdots \\
C A^{M-2} \\
C A^{M-1}
\end{array}\right], \\
N \triangleq\left[\begin{array}{ccccc}
0 & 0 & \cdots & 0 & 0 \\
C G & 0 & \cdots & 0 & 0 \\
C A G & C G & \cdots & 0 & 0 \\
\vdots & \vdots & \vdots & \vdots & \vdots \\
C A^{M-2} G & C A^{M-3} G & \cdots & C G & 0
\end{array}\right] .
\end{gathered}
$$

Using finite measurements $Z(i)$ on the most recent window $[i-M, i]$, FMS smoothers [6-10] as well as FMS filters [1215] have been developed.

\section{Alternative FMS Smoother with Forgetting Factor}

In this paper, the fixed-lagged system state $x(i-d)$ at the time $i-d$ is considered. As shown in [6-10], the fixedlagged system state means there is a fixed delay between the measurement and the availability of its estimate. The delay length $d$ is the positive integer with $0 \leq d<M$. This delay length means the number of discrete time steps between the lagged time $i-d$ when the state is to be estimated and the current time $i$. To estimate fixed-lagged system state $x(i-$ $d)$, an alternative FMS smoother is developed using finite measurements $Z(i)$ on the most recent window $[i-M, i]$.

As shown in [6-10], the state $x(i-d)$ at the lagged time $i-d$ is represented from the discrete time state-space model (1) as follows:

$$
x(i-d)=A^{M-d} x(i-M)+\widetilde{N} W(i)
$$

where

$$
\widetilde{N} \triangleq\left[\begin{array}{llllllll}
A^{M-d-1} G & \cdots & \left.\begin{array}{lllll}
0 & d & & & \\
0 & 0 & \cdots & 0
\end{array}\right]
\end{array}\right]
$$

Therefore, finite measurements $Z(i)$ (3) can be modified by

$$
Z(i)=\bar{L} x(i-d)+\bar{N} W(i)+V(i)
$$

where

$$
\begin{aligned}
& \bar{L} \triangleq L A^{-(M-d)}, \\
& \bar{N} \triangleq N-L A^{-(M-d)} \widetilde{N} .
\end{aligned}
$$

An alternative FMS smoother is developed under a least squares criterion using the forgetting factor strategy. Given measurements $Z(i)$ on the most recent window $[i-M, i]$, the FMS smoother $\widehat{x}(i-d)$ is obtained from the following forgetting factor least squares criterion:

$$
\widehat{x}(i-d)=\arg \min _{x(i-d)}[Z(i)-\bar{L} x(i-d)]^{T} \Lambda[Z(i)-\bar{L} x(i-d)],
$$

where $\Lambda$ is a diagonal matrix as

$$
\Lambda \triangleq \operatorname{diag}\left[\begin{array}{llll}
\lambda^{M-1} & \lambda^{M-2} & \cdots & \lambda^{0}
\end{array}\right], \quad 0<\lambda \leq 1,
$$

where $\lambda$ is called the forgetting factor. The forgetting factor strategy has been well-known in estimation areas to give exponentially less weight to older error samples as shown in [16-18]. A main role of the forgetting factor $\lambda$ is to account for the fact that the state-space models (1) are not perfect to globally model the observed phenomenon and thus is to make the model that is locally well modeling the observations by concentrating on finite measurements on the most recent 
window $[i-M, i]$. Then, when $\{A, C\}$ is observable and $M \geq n$, the solution of (9) is given by the following matrix form:

$$
\begin{aligned}
\widehat{x}(i-d) & =\left(\bar{L}^{T} \Lambda^{T / 2} \Lambda^{1 / 2} \bar{L}\right)^{-1} \bar{L}^{T} \Lambda^{T / 2} \Lambda^{1 / 2} Z(i) \\
& =\Sigma \Gamma^{T} \Lambda^{1 / 2} Z(i)
\end{aligned}
$$

where

$$
\begin{gathered}
\Sigma \triangleq A^{M}\left(\Gamma^{T} \Gamma\right)^{-1}, \\
\Gamma \triangleq \Lambda^{1 / 2} L A^{d}=\left[\begin{array}{c}
\check{\Gamma} \\
C A^{M+d-1}
\end{array}\right]=\left[\begin{array}{c}
\lambda^{(M-1) / 2} C A^{d} \\
\lambda^{-1 / 2} \check{\Gamma} A
\end{array}\right], \\
\widetilde{Z}(i) \triangleq \Lambda^{1 / 2} Z(i)=\left[\begin{array}{c}
\lambda^{(M-1) / 2} z(i-M) \\
\check{Z}(i)
\end{array}\right],
\end{gathered}
$$

where $\check{\Gamma}$ is upper $M-1$ rows of $\Gamma$ and $\check{Z}(i)$ is lower $M-1$ rows of $\widetilde{Z}(i)$.

In the following theorem, the proposed FMS smoother (11) with a matrix form is represented in a recursive form.

Theorem 1. When $\{A, C\}$ is observable and $M \geq n$, the FMS smoother $\widehat{x}(i-d)$ (11) can be represented in the following recursive form:

$$
\widehat{x}(i-d+1)=\mathscr{F} \hat{x}(i-d)+\mathscr{H}_{1} z(i-M)+\mathscr{H}_{2} z(i),
$$

where smoother gain matrices are defined by

$$
\begin{aligned}
& \mathscr{F} \triangleq \lambda \Sigma\left(\Sigma A^{T}\right)^{-1}, \\
& \mathscr{H}_{1} \triangleq-\lambda^{M} \Sigma\left(C A^{d-1}\right)^{T}, \\
& \mathscr{H}_{2} \triangleq \Sigma\left(C A^{M+d-1}\right)^{T} .
\end{aligned}
$$

Proof. Using (12), at the lagged time $i-d$, the FMS smoother $\widehat{x}(i-d)(11)$ can be written by

$$
\begin{aligned}
\widehat{x}(i-d)= & \Sigma\left[\begin{array}{c}
\lambda^{(M-1) / 2} C A^{d} \\
\lambda^{-1 / 2} \check{\Gamma} A
\end{array}\right]^{T}\left[\begin{array}{c}
\lambda^{(M-1) / 2} z(i-M) \\
\check{Z}(i)
\end{array}\right] \\
= & \lambda^{M-1} \Sigma\left(C A^{d}\right)^{T} z(i-M) \\
& +\lambda^{-1 / 2} \Sigma A^{T} \check{\Gamma}^{T} \check{Z}(i) .
\end{aligned}
$$

Using (15), at the lagged time $i-d+1$, the FMS smoother $\widehat{x}(i-d+1)$ can be written by

$$
\begin{aligned}
\widehat{x}(i-d+1) & =\Sigma \Gamma^{T} \Lambda^{1 / 2} Z(i+1) \\
& =\Sigma\left[\begin{array}{c}
\check{\Gamma} \\
C A^{M+d-1}
\end{array}\right]^{T}\left[\begin{array}{c}
\lambda^{1 / 2} \check{Z}(i) \\
z(i)
\end{array}\right] \\
& =\lambda^{1 / 2} \Sigma \check{\Gamma}^{T} \check{Z}(i)+\Sigma\left(C A^{M+d-1}\right)^{T} z(i)
\end{aligned}
$$

$$
\begin{aligned}
= & \lambda \Sigma\left(\sum A^{T}\right)^{-1} \widehat{x}(i-d) \\
& -\lambda^{M} \Sigma\left(C A^{d-1}\right)^{T} z(i-M) \\
& +\Sigma\left(C A^{M+d-1}\right)^{T} z(i) .
\end{aligned}
$$

This completes the proof.

Note that this recursive form has not been developed by the existing FMS smoother in [6-10]. Smoother gain matrices $\mathscr{F}, \mathscr{H}_{1}$, and $\mathscr{H}_{2}$ in (14) require offline computations only on the interval $[0, M]$ once and then they are time-invariant for all windows. Therefore, the proposed FMS smoother can be time-invariant.

In the following theorem, the proposed FMS smoothers in (11) and (13) are shown to have an unbiasedness property when there are noises and a deadbeat property when there are no noises. The unbiasedness property means that the mean value of $\widehat{x}(i-d)$ tracks the mean value of the state at every time. The deadbeat property means that $\widehat{x}(i-d)$ tracks exactly the state at every time.

Theorem 2. When $\{A, C\}$ is observable and $M \geq n$, the FMS smoother $\widehat{x}(i-d)$ is unbiased for noisy systems and exact for noise-free systems.

Proof. When there are noises on the window $[i-M, i]$, since $\bar{N} W(i)+V(i)$ is zero-mean in $(7), E[Z(i)]=\bar{L} E[x(i-d)]=$ $L A^{-(M-d)} E[x(i-d)]$. Therefore, the following is obtained from (11):

$$
\begin{aligned}
E[\widehat{x}(i-d)] & =\Sigma \Gamma^{T} \Lambda^{1 / 2} E[Z(i)] \\
& =\Sigma \Gamma^{T} \Lambda^{1 / 2} L A^{d} A^{-M} E[x(i-d)] \\
& =\Sigma \Gamma^{T} \Gamma A^{-M} E[x(i-d)] \\
& =\Sigma \Sigma^{-1} E[x(i-d)]=E[x(i-d)] .
\end{aligned}
$$

This completes the proof of the unbiasedness property.

When there are no noises on the window $[i-M, i]$ as $x(i+1)=A x(i)$ and $z(i)=C x(i)$, the observation $Z(i)$ is determined by the current state $x(i-d)$ as $Z(i)=\bar{L} x(i-d)=$ $L A^{d} A^{-M} x(i-d)$. Therefore, the following is obtained directly from the proof of the unbiasedness property:

$$
\widehat{x}(i-d)=x(i-d) \text {. }
$$

This completes the proof of the deadbeat property.

The deadbeat property in Theorem 2 means that the FMS smoother designed for the system (1) with noises provides exact state when, in actual, there are no noises. Note that the proposed FMS smoother can have the finite convergence time and the fast estimation ability due to the deadbeat property. Hence, the proposed FMS smoother can be useful in many engineering problems such as fault detection and diagnosis, maneuver detection, and target tracking, because these 
problems require fast estimation and detection of signals with unknown times of occurrence. These good inherent properties, time-invariance, unbiasedness, and deadbeat of the proposed FMS smoother cannot be obtained by the IMS smoother such as the Kalman smoother.

The important issue here is how to choose an appropriate forgetting factor $\lambda$ that makes the residual performance as good as possible. When the window length $M$ is fixed, the forgetting factor $\lambda$ should be chosen. Intuitively, a reasonable criterion for the choice of the forgetting factor should be how much information about the current state of the system the older data and the new data contain. If the newly coming data bring enough information about the current state, or the older data contain less information on the present data, the forgetting factor $\lambda$ should be smaller. When the exact information about the noise covariances cannot be obtained but some rough information about the noises can be obtained, there are some choices of the forgetting factor. If the covariance $Q$ of the system noise $w(i)$ is smaller, the older data should contain more information on the current state. Therefore, the smaller $Q$ is, the bigger forgetting factor $\lambda$ should be. In comparison with the covariance $Q$ of the system noise, if the covariance $R$ of the measurement noise $v(i)$ is relatively bigger, $\lambda$ should be bigger too. Intuitively, the above facts can be roughly explained as follows. When the covariance $R$ of the measurement noise is larger, more data should be used to suppress the influence of the noise by means of averaging the measurement data, which means that the forgetting factor $\lambda$ should be larger. Thus, the forgetting factor $\lambda$ is a continuous parameter to adjust finely the smoothing performance.

\section{Computer Simulations}

Computer simulations are performed for the following discrete time F-404 engine system [19] with a fixed-lag $d$ as well as a uncertain model parameter $\delta(i)$ to illustrate the validity of the proposed FMS smoother and to compare with both existing FMS smoother and IMS smoother

$$
\begin{aligned}
& A=\left[\begin{array}{ccc}
0.9305+\delta(i) & 0 & 0.1107 \\
0.0077 & 0.9802+\delta(i) & -0.0173 \\
0.0142 & 0 & 0.8953+\delta(i)
\end{array}\right], \\
& G=\left[\begin{array}{l}
1 \\
1 \\
1
\end{array}\right] \\
& C=\left[\begin{array}{lll}
1 & 0 & 0 \\
0 & 1 & 0
\end{array}\right]
\end{aligned}
$$

where actual system and measurement noise covariances are taken as $Q=0.2^{2}$ and $R=0.4^{2}$. The delay length is set by $d=4$.

First, to show how to choose the forgetting factor $\lambda$, the proposed FMS smoother is compared with the existing FMS smoother. To make a clearer comparison of estimation
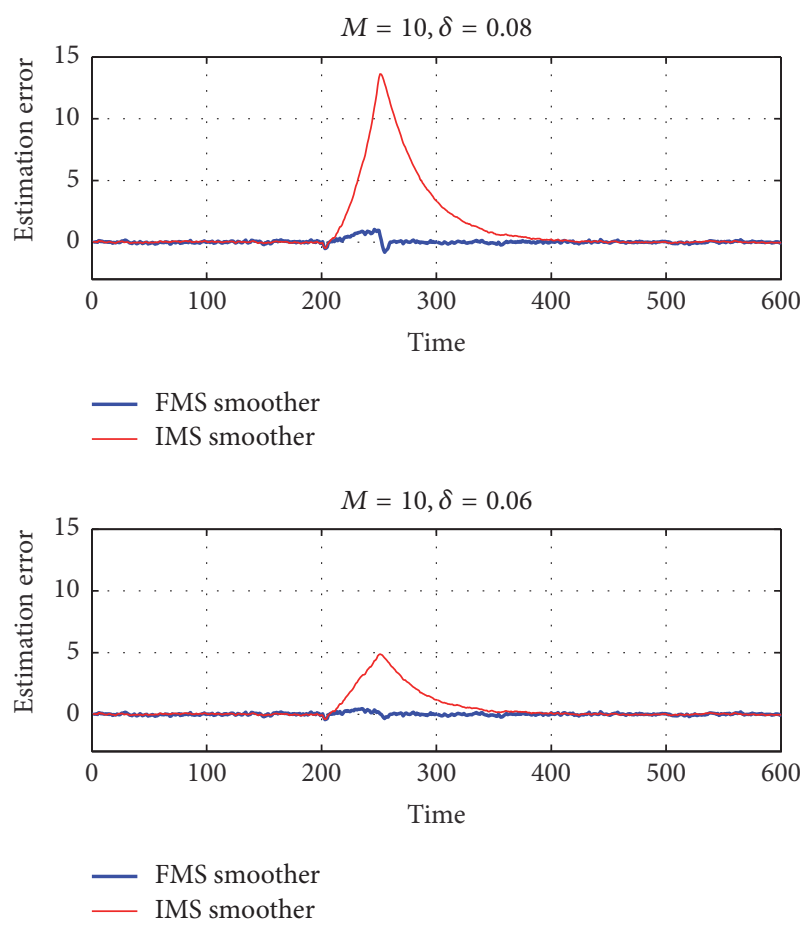

FIGURE 1: Estimation error for temporary modeling uncertainty when $M=10$.

performances, Monte Carlo simulations of 100 runs are performed and in each simulation 600 sample points are generated. The estimation performance of smoothers can be evaluated by the mean of root-squared estimation error. It was mentioned in previous section that when the measurement noise covariance $R$ is relatively bigger than the system noise covariance $Q, \lambda$ should be bigger too. It is easy to see that the above states seem right from simulation results in Table 1 when the window length is taken $M=20$. These results can provide practical guidance on the choice of a forgetting factor $\lambda$. The simulation results in Table 2 show estimation performance of the proposed FMS smoother with $\lambda=0.9$ and the existing FMS smoother. In comparison with the existing FMS smoother, the FMS smoother performs worse than when information about noise covariances is assumed to be exactly known. This may be the expected result since the existing FMS smoother in this case is optimal. However, when this assumption is not satisfied, the performance of the FMS smoother can be better than that of the existing FMS smoother.

Secondly, the proposed FMS smoother and the fixedlag Kalman smoother are compared for the temporarily uncertain system. The uncertain model parameter is taken by two cases, $\delta(i)=0.8$ and $\delta(i)=0.6$ for the interval $200 \leq i \leq 250$ the temporarily uncertain F404 engine system (19). In addition, the window length is taken by two cases, $M=10$ and $M=20$ for the proposed FMS smoother. As shown in Figures 1 and 2, the estimation error of the proposed FMS smoother is smaller than that of the fixed-lag Kalman smoother on the interval where modeling uncertainty exists for all cases. In addition, the convergence 
TABLE 1: Mean of root-squared estimation error of the proposed FMS smoother for various $\lambda \mathrm{s}$.

\begin{tabular}{lccccc}
\hline$\lambda$ & 0.7 & 0.8 & 0.9 & 0.95 & 1 \\
\hline$M=10$ & 0.1403 & 0.1070 & 0.0411 & 0.0269 & 0.0747 \\
\hline
\end{tabular}

TABLE 2: Mean of root-squared estimation error of two FMS smoothers.

\begin{tabular}{|c|c|c|c|}
\hline & \multicolumn{2}{|c|}{ Existing FMS smoother } & \multirow{2}{*}{$\begin{array}{l}\text { Proposed FMS smoother } \\
\qquad \lambda=0.95\end{array}$} \\
\hline & $\begin{array}{c}\text { Correct } \\
Q=0.5^{2}, R=1^{2}\end{array}$ & $\begin{array}{c}\text { Incorrect } \\
Q=2^{2}, R=1^{2}\end{array}$ & \\
\hline$M=10$ & 0.0054 & 0.1824 & 0.0269 \\
\hline
\end{tabular}

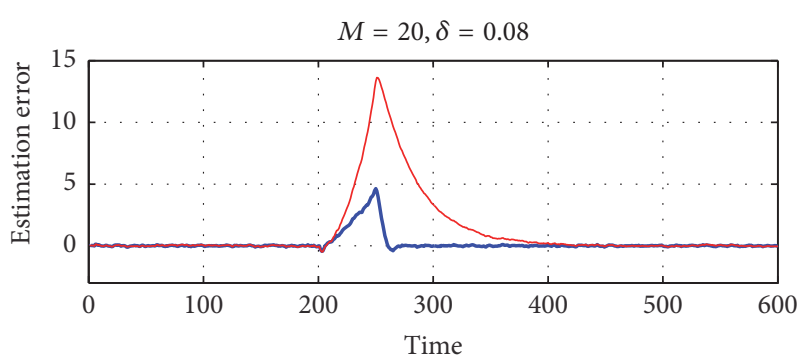

— FMS smoother _ IMS smoother

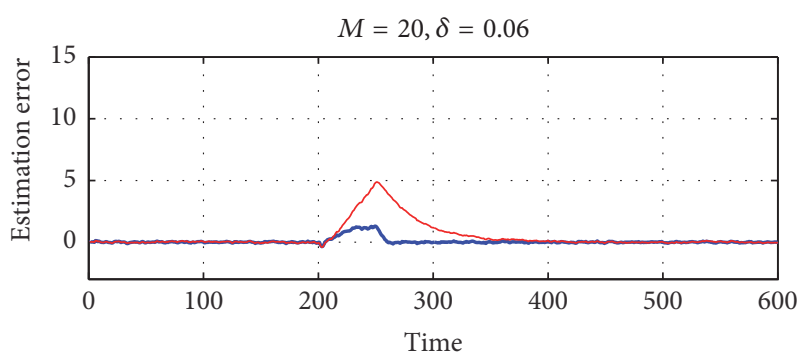

- FMS smoother

FIGURE 2: Estimation error for temporary modeling uncertainty when $M=20$.

of estimation error is much faster than that of the fixedlag Kalman smoother after temporary modeling uncertainty disappears. This phenomenon is more remarkable when the model is more uncertain; that is, $\delta(i)=0.8$. Of course, the fixed-lag Kalman smoother can outperform the proposed FMS smoother after the effect of temporary modeling uncertainty completely disappears. Therefore, the proposed FMS smoother can be more robust than the fixed-lag Kalman smoother when applied to temporarily uncertain systems, although the proposed FMS smoother is designed with no consideration for robustness. Moreover, it can be known that a large window length may yield the long convergence time of the estimation error and thus degrades the performance of the the proposed FMS smoother. Therefore, it can be stated that the window length $M$ can be considered as a useful parameter to make the performance of the the proposed FMS smoother as good as possible.

\section{Conclusions}

In this paper, an alternative FMS smoother with a recursive form has been proposed under a least squares criterion using a forgetting factor strategy. The proposed FMS smoother does not require information of the noise covariances as well as the initial state. The proposed FMS smoother has been shown to have good inherent properties such as time-invariance, unbiasedness, and deadbeat. The forgetting factor has been shown to be considered as useful parameter to make the estimation performance of the proposed FMS smoother as good as possible. It has been shown from computer simulations that the proposed FMS smoother can be better than the existing FMS smoother for incorrect noise covariances and the IMS smoother for temporary uncertainties.

As an alternative design parameter for the proposed FMS smother, the delay length $d$ should be considered in future works. In addition, the combination with the window length and the forgetting factor should be also researched to improve smoothing performance.

\section{Conflicts of Interest}

The author declared no potential conflicts of interest with respect to the research, authorship, and/or publication of this article.

\section{References}

[1] N. Morrison, Introduction to Sequential Smoothing and Prediction, vol. 4, McGraw Hill, 1st edition edition, 1969.

[2] M. S. Grewal and A. P. Anderews, Kalman filtering: theory and Practice, Prentice-Hall, Englewood Cliffs, NJ, USA, 1993.

[3] S. Challa, R. J. Evans, X. Wang, and J. Legg, "A fixed-lag smoothing solution to out-of-sequence information fusion problems," Communications in Information and Systems, vol. 2, no. 4, pp. 325-348, 2002.

[4] L. Mirkin and G. Tadmor, "Fixed-lag smoothing as a constrained version of the fixed-interval case," Proc. American Control Conference, pp. 4166-4170, 2004.

[5] T. L. Ogle and W. D. Blair, "Fixed-lag alpha-beta filter for target trajectory smoothing," IEEE Transactions on Aerospace and Electronic Systems, vol. 40, no. 4, pp. 1417-1421, 2004.

[6] B. K. Kwon, S. Han, O. K. Kwon, and W. H. Kwon, "Minimum variance FIR smoothers for discrete-time state space models," IEEE Signal Processing Letters, vol. 14, no. 8, pp. 557-560, 2007. 
[7] P.-S. Kim and J. H. Choi, "A new fixed-lag smoother using recent finite observations," in Proceedings of the 7th International Conference on Information, Communications and Signal Processing, ICICS 2009, pp. 1-5, Macau, China, December 2009.

[8] Y. S. Shmaliy and L. J. Morales-Mendoza, "FIR smoothing of discrete-time polynomial signals in state space," IEEE Transactions on Signal Processing, vol. 58, no. 5, pp. 2544-2555, 2010.

[9] P. S. Kim, "A computationally efficient fixed-lag smoother using recent finite measurements," Measurement: Journal of the International Measurement Confederation, vol. 46, no. 1, pp. 846-850, 2013.

[10] P. S. Kim, M. S. Jang, and E. H. Lee, "A discrete time-varying finite memory smoother and its application in fault detection," ICIC Express Letters-An International Journal of Research and Surveys, vol. 8, no. 4, pp. 957-962, 2014.

[11] R. J. Fitzgerald, "Divergence of the Kalman Filter," IEEE Transactions on Automatic Control, vol. 16, no. 6, pp. 736-747, 1971.

[12] P.-S. Kim, "An alternative FIR filter for state estimation in discrete-time systems," Digital Signal Processing: A Review Journal, vol. 20, no. 3, pp. 935-943, 2010.

[13] S. Zhao, Y. S. Shmaliy, B. Huang, and F. Liu, "Minimum variance unbiased FIR filter for discrete time-variant systems," Automatica, vol. 53, no. 2, pp. 355-361, 2015.

[14] Y. S. Shmaliy, S. Khan, and S. Zhao, "Ultimate iterative UFIR filtering algorithm," Measurement: Journal of the International Measurement Confederation, vol. 92, pp. 236-242, 2016.

[15] P. S. Kim, E. H. Lee, M. S. Jang, and S. Y. Kang, "A finite memory structure filtering for indoor positioning in wireless sensor networks with measurement delay," International Journal of Distributed Sensor Networks, vol. 13, no. 1, pp. 1-8, 2017.

[16] A. Vahidi, A. Stefanopoulou, and H. Peng, "Recursive least squares with forgetting for online estimation of vehicle mass and road grade: theory and experiments," Vehicle System Dynamics, vol. 43, no. 1, pp. 31-55, 2005.

[17] P.-S. Kim and M.-E. Lee, "A new FIR filter for state estimation and its application," Journal of Computer Science and Technology, vol. 22, no. 5, pp. 779-784, 2007.

[18] C. Paleologu, J. Benesty, and S. Ciochiňa, "A robust variable forgetting factor recursive least-squares algorithm for system identification," IEEE Signal Processing Letters, vol. 15, no. 3, pp. 597-600, 2008.

[19] R. W. Eustace, B. A. Woodyatt, G. L. Merrington, and A. Runacres, "Fault signatures obtained from fault implant tests on an F404 engine," Journal of Engineering for Gas Turbines and Power, vol. 116, no. 1, pp. 178-183, 1994. 


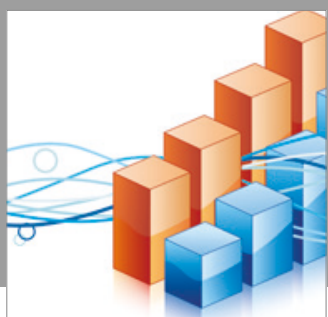

Advances in

Operations Research

vatersals

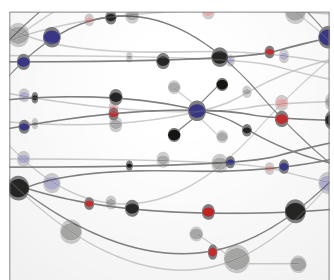

\section{The Scientific} World Journal
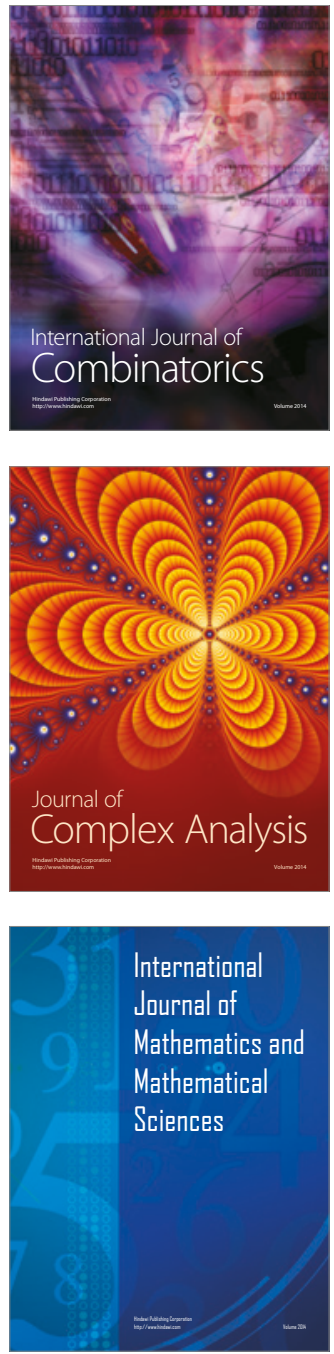
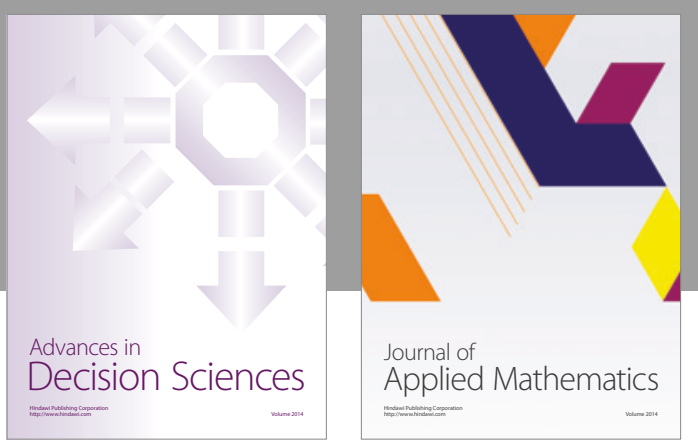

Algebra

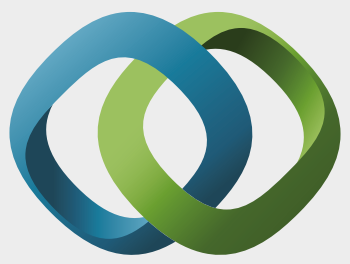

\section{Hindawi}

Submit your manuscripts at

https://www.hindawi.com
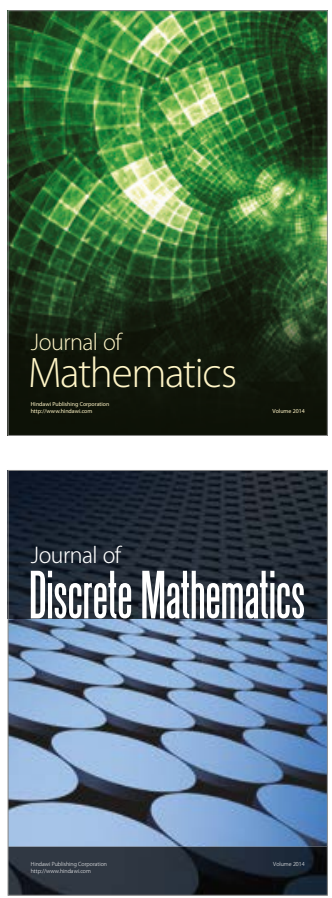

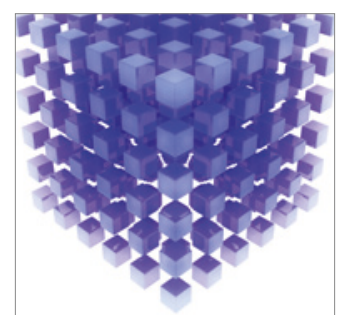

Mathematical Problems in Engineering
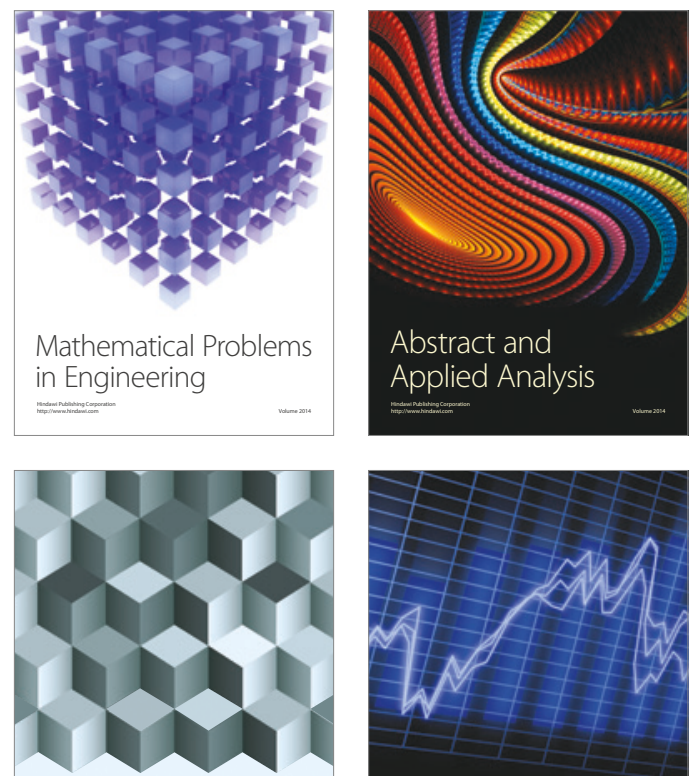

Journal of

Function Spaces

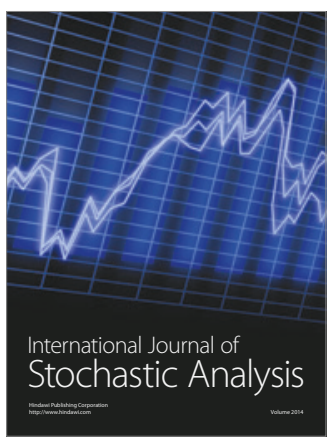

Probability and Statistics
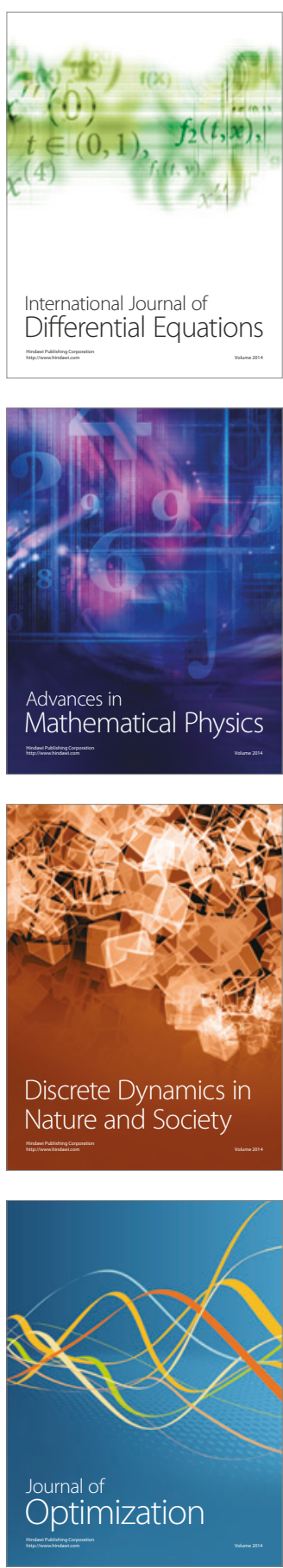